\title{
D.C. conductivity and spectroscopic studies of polyaniline doped with binary dopant $\mathrm{ZrOCl}_{2} / \mathrm{AgI}$
}

\author{
KIRAN KUMARI, VAZID ALI*, ANAND KUMAR ${ }^{\dagger}$, SUSHIL KUMAR ${ }^{\dagger}$ and M ZULFEQUAR ${ }^{\dagger \dagger}$ \\ Department of Chemistry, Chaudhary Devi Lal University, Sirsa 125 055, India \\ ${ }^{\dagger}$ Department of Physics, Chaudhary Devi Lal University, Sirsa 125 055, India \\ ${ }^{\dagger}$ Department of Physics, Jamia Millia Islamia (Central University), New Delhi 110 025, India
}

MS received 24 December 2010; revised 10 March 2011

\begin{abstract}
Aqueous binary dopant ( $\left.\mathrm{ZrOCl}_{2} / \mathrm{AgI}\right)$ is used in different ratios such as 1:1, 1:2 and 2:1 (w/w) for chemical doping to enhance the conductivity of synthesized polyaniline (PANI). The doping of polyaniline is carried out using tetrahydrofuran as a solvent. Doped samples are characterized using various techniques such as $I-V$ characteristics, UV-visible spectroscopy, X-ray diffractometry (XRD), FTIR and photoluminescence (PL) studies. A significant enhancement in d.c. conductivity has been observed with the introduction of binary dopant. UV-visible study shows that optical parameters change considerably after doping. Interestingly, both direct and indirect bandgaps are observed in the doped samples. XRD patterns show the semi-crystalline nature of doped polyaniline. FTIR study shows structural modifications in functional groups with doping in PANI. Photoluminescence spectra exhibit emission properties of the samples.
\end{abstract}

Keywords. Polyaniline; D.C. conductivity; UV-visible; XRD; FTIR; PL.

\section{Introduction}

Polymers are typically utilized in electrical, optical and electronic devices as insulators because of their very high electrical resistivity. The dielectric properties of heterogeneous polymers (Planes et al 1998) play an important role in device applications such as high performance capacitors, electrical cable insulation, electronic packaging, components etc. Polymers are usually polyconjugated structures which are insulators in their pure state; but when treated with oxidizing or reducing agents they can be converted into polymer salts having reasonable electrical conductivity. Conjugated polymers are plastic semiconductors (Friend et al 1997). They have wide applications in devices such as solar cells, rechargeable batteries, light emitting diodes, microactuators, electrochromic displays, field effect transistors, sensors etc (Saraswathi et al 1999).

In polymers, doping can be carried out by different processes such as chemical and electrochemical. Recently, metal salt doping in polyaniline has been reported (Ali et al 2006). Among these polymers, polyaniline has attracted much attention of many researchers due to its ease of synthesis, processibility, good thermal stability and good environmental stability. MacDiarmid et al (1984) investigated polyaniline as an electrically conducting polymer, which is emerging as a promising synthetic metal. The possibility of synthesizing and doping of polyaniline with protonic acid dopants containing different types of counterions is one of the key

*Author for correspondence (akdmdu@gmail.com) factors responsible for the versatility of this class of polymers. Photoluminescent organic molecules are a new class of compounds with interesting properties. They undergo emission over a wide range from violet to red. They can also be combined in several different forms to produce white light. One category of organic material with photoluminescence properties is conjugated organic polymers.

In the present work, our approach is to study the binary metal salt $\left(\mathrm{ZrOCl}_{2} / \mathrm{AgI}\right)$ induced chemical doping in polyaniline in the presence of distilled water and tetrahydrofuran. The reason for choosing the combination of $\mathrm{ZrOCl}_{2}$ and $\mathrm{AgI}$ as binary dopant is that $\mathrm{ZrOCl}_{2}$ is a luminescent material contributing towards photoluminescence; while $\mathrm{AgI}$ is a conducting material and hence contributing towards d.c. conductivity. Therefore, by choosing such a combination, we can prepare a material having good conductivity as well as photoluminescence simultaneously. Sincere efforts have been made to understand the effect of binary dopant on the electrical and spectroscopic properties of polyaniline using $I-V$ characteristics method, UV-visible spectroscopy, $\mathrm{X}$ ray diffractometry (XRD), and FTIR and photoluminescence (PL) studies.

\section{Experimental}

\subsection{Chemicals}

Aniline (Loba Chemicals, 99\% purity), potassium dichromate (S.D. Fine Chemicals, A.R. grade), hydrochloric acid 
(Qualigens Fine Chemicals, A.R. grade), ammonia solution in water $28 \%$ (S.D. Fine Chemicals), tetrahydrofuran (Merck India, A.R. grade), $\mathrm{ZrOCl}_{2}$ (S.D. Fine Chemical, A.R. grade), AgI (Hi-Media Lab., A.R. grade) were used. The binary dopant $\left(\mathrm{ZrOCl}_{2} / \mathrm{AgI}\right)$ was prepared by mixing their powders homogeneously taken as per their (w/w) ratio $(1: 1,1: 2,2: 1)$.

\subsection{Synthesis and chemical doping}

Distilled aniline was used to synthesize polyaniline (PANI) by chemical oxidation polymerization in acidic medium as suggested by MacDiarmid et al (1986). Synthesized polyaniline (PANI) was dried in an oven and grinded to obtain PANI powder. Three systems of binary dopant $\left(\mathrm{ZrOCl}_{2} / \mathrm{AgI}\right)$ having ratios 1:1, 1:2 and 2:1 (w/w) were used. 2.0 g PANI powder and dopant having $2 \%(\mathrm{w} / \mathrm{w})$ concentration were used in $10 \mathrm{ml}$ tetrahydrofuran (THF) solvent with magnetic stirring for about $15 \mathrm{~min}$, and then kept in an oven at $30^{\circ} \mathrm{C}$ for $24 \mathrm{~h}$ to perform doping process completely in polyaniline. The physical state of binary dopant, $\mathrm{ZrOCl}_{2} / \mathrm{AgI}$, was solid particles in suspension which appeared milky in tetrahydrofuran (THF). Subsequently, chemically doped polyaniline was put in an oven at $110^{\circ} \mathrm{C}$ for $4 \mathrm{~h}$ to achieve moisture free doped polyaniline.

\section{Results and discussion}

\subsection{D.C. conductivity}

D.C. conductivity of undoped and $\left(\mathrm{ZrOCl}_{2} / \mathrm{AgI}\right)$ doped polyaniline of the pellets (diameter, $1.01 \mathrm{~cm}$, thickness, $0.016 \mathrm{~cm}$ ) was measured by using two-probe method at a temperature of 298 K. D.C. conductivity of the pellets was measured by mounting them between steel electrodes inside a specially designed sample holder. The temperature was measured with a calibrated copper-constantan thermocouple mounted near the electrodes. The samples were annealed to avoid any effect of moisture absorption. These measurements were made at a pressure of about $10^{-3}$ Torr. A stabilized voltage of $1.5 \mathrm{~V}$ was applied across the sample and the resultant current was measured with a pico-ammeter, which gives d.c. conductivity within $\pm 1 \%$ of accuracy (Majeed Khan et al 2004). Conductivity was measured by using Ohm's law,

$$
V=R I,
$$

where $I$ is the current (in amperes) through a resistor, $R$ (in ohms) and $V$ the drop in potential (in volts) across it. The reciprocal of resistance $\left(R^{-1}\right)$ is called conductance, the flow of current, $I$, as a result of gradient in potential leads to energy being dissipated $\left(R I^{2}\right.$ joule $\left.\mathrm{s}^{-1}\right)$.

In ohmic material, the resistivity measured is proportional to the sample cross-section, $A$, and inversely proportional to its length, $l$ :

$$
R=\rho l / A,
$$

where $\rho$ is the resistivity $(\Omega \mathrm{cm})$. Its inverse $\sigma=\rho^{-1}$ is the conductivity $\left(\Omega^{-1} \mathrm{~cm}^{-1}\right)$. It is found that d.c. conductivity of $\left(\mathrm{ZrOCl}_{2} / \mathrm{AgI}\right)$ doped PANI samples changes on changing their ratio, and is shown in table 1.

D.C. conductivity of pure PANI increases exponentially with temperature, exhibiting semiconductor behaviour. The doping of conducting polymers implies charge transfer, the associated insertion of a counter ion and the simultaneous control of Fermi level or chemical potential. The electrical conductivity of conducting polymers results from mobile charge carriers introduced into $\pi$-electronic system through doping. At low doping levels these charge carriers are selflocalized and form non-linear configuration. Because of large interchain transfer integrals, the transport of charge is believed to be principally along the conjugated chains, with interchain hopping as a necessary secondary condition (Scrosati 1993). In PANI, there are nearly degenerate ground states, the dominating charge carriers are polarons and bipolarons. It is observed that $\mathrm{ZrOCl}_{2} / \mathrm{AgI}$ doped PANI shows charge carriers formation with linear configuration; as a result conductivity changes substantially.

\subsection{Optical studies}

UV-visible study of doped polyaniline samples is performed using a spectrophotometer (Perkin Elmer Lambda). Optical parameters such as absorption coefficient $(\alpha)$, extinction coefficient $(k)$ and energy bandgap $\left(E_{\mathrm{g}}\right)$ have been determined for undoped and $\left(\mathrm{ZrOCl}_{2} / \mathrm{AgI}\right)$ ratios 1:1, 1:2, 2:1

Table 1. Optical parameters and D.C. conductivity of polyaniline doped with $\left(\mathrm{ZrOCl}_{2} / \mathrm{AgI}\right)$ doping system ratio (1:1, 1:2 and 2:1 (w/w))

\begin{tabular}{|c|c|c|c|c|c|c|}
\hline $\begin{array}{l}\text { Ratio of } \\
\left(\mathrm{ZrOCl}_{2} / \mathrm{AgI}\right) \\
(\mathrm{w} / \mathrm{w})\end{array}$ & $\begin{array}{c}\text { Dopant }\left(\mathrm{ZrOCl}_{2} / \mathrm{AgI}\right) \\
\text { conc. in polyaniline } \\
(\mathrm{w} / \mathrm{w})\end{array}$ & $\begin{array}{l}\text { Direct bandgap, } \\
E_{\mathrm{g}}(\mathrm{eV})\end{array}$ & $\begin{array}{c}\text { Indirect bandgap, } \\
E_{\mathrm{g}}(\mathrm{eV})\end{array}$ & $\begin{array}{l}\text { Absorption coeff. } \alpha \\
\text { at } \lambda=400 \mathrm{~nm}\end{array}$ & $\begin{array}{l}\text { Extinction coeff. } k \\
\text { at } \lambda=400 \mathrm{~nm}\end{array}$ & $\begin{array}{c}\text { D.C. conductivity, } \\
\sigma(\mathrm{S} / \mathrm{cm})\end{array}$ \\
\hline & Undoped & 2.74 & 1.82 & 0.99 & 31.81 & $0.66 \times 10^{-8}$ \\
\hline $1: 1$ & $2 \%$ doped & 1.43 & 1.44 & 1.49 & 47.72 & $0.72 \times 10^{-5}$ \\
\hline $1: 2$ & $2 \%$ doped & 1.35 & 1.40 & 1.39 & $44 \cdot 54$ & $0.67 \times 10^{-5}$ \\
\hline $2: 1$ & $2 \%$ doped & $1 \cdot 37$ & $1 \cdot 38$ & $0 \cdot 89$ & $28 \cdot 63$ & $0.77 \times 10^{-5}$ \\
\hline
\end{tabular}
at $2 \%$ dopant concentration. 
(w/w) doped polyaniline samples at $298 \mathrm{~K}$ through absorption spectra. The relationship between the optical bandgap, absorption coefficient and incident photon energy $(h v)$ is given by (Gosain et al 1991; Epstein et al 1994)

$$
\alpha h v \propto\left(h v-E_{\mathrm{g}}\right)^{n},
$$

where $n=1 / 2,3 / 2, \ldots$ for direct and $n=1,2,3, \ldots$. for indirect transitions, respectively.

It has been observed that both undoped and doped polyaniline samples show direct and indirect transitions. The direct and indirect bandgaps both exhibit decreasing orders. The extinction coefficient is determined by the following relation

$$
k=\alpha \lambda / 4 \pi \text {. }
$$

Absorption coefficient and extinction coefficient have been determined using sharp increase of absorption spectra at a wavelength of $400 \mathrm{~nm}$ as shown in figure 1. There are rising bends/curves at a wavelength of $380 \mathrm{~nm}$, which are attributed to oxidized phase of polyaniline, another moderate peak at $\sim 400 \mathrm{~nm}$ appears for all studied samples. It has also been observed in $\left(\mathrm{ZrOCl}_{2} / \mathrm{AgI}\right)$ doped polyaniline sample that there is a change in bandgap at different ratios 1:1, 1:2 and 2:1 (w/w). Absorption coefficient and extinction coefficient also changes with changing ratio. Conclusively, $\left(\mathrm{ZrOCl}_{2} / \mathrm{AgI}\right)$ plays a significant role in the chemical doping of polyaniline and the measured optical parameters show significant changes. Thus, information about the changes in optical parameters by chemical doping with $\left(\mathrm{ZrOCl}_{2} / \mathrm{AgI}\right)$ may explore the possibilities in the course of development with new metal salts systems in conducting polymers.

\subsection{XRD studies}

XRD patterns provide information in relation to the nature and structure of the samples. XRD pattern of undoped sample of polyaniline shows amorphous nature. XRD patterns of doped samples show semi-crystalline nature. Since the conductivity of polymers depends on various parameters such as doping level (carrier's concentration), formation of polarons and bipolarons (Fink and Leising 1986), the semicrystalline nature of polymers arises owing to the systematic alignment of polymer chain folding or by the formation of single or multiple helices, for part of their length (Kazim et al 2006). XRD patterns of undoped and doped polyaniline with $2 \%$ doping concentration and binary dopant ratios 1:1, 1:2, 2:1 are shown in figure $2(\mathrm{a}-\mathrm{d})$. XRD pattern of undoped PANI sample shows an amorphous hump around $20^{\circ}$ (figure 2a) and the doped samples have a peak at $4^{\circ}$ (figure $2 \mathrm{~b}$ ). As shown in figure 2(c), broadening of peaks in XRD pattern have been obtained at about $27.5^{\circ}$ corresponding to a ratio 1:2 of binary dopant. In $2: 1$ doped sample there are three small peaks at $21^{\circ}, 23^{\circ}$ and $39^{\circ}$ (figure $2 \mathrm{~d}$ ). The variation in diffraction intensity with binary dopant ratio exhibits the interaction of dopant with PANI network.

\section{$3.4 \quad$ FTIR studies}

FTIR spectra have been recorded for undoped and doped PANI (2\% doped) with binary dopant $\left(\mathrm{ZrOCl}_{2} / \mathrm{AgI}\right)$ ratios of $1: 1,1: 2$ and $2: 1(\mathrm{w} / \mathrm{w})$ and are shown in figure $3(\mathrm{a}-\mathrm{d})$. The broad medium band at $3140 \mathrm{~cm}^{-1}$ in $1: 1\left(\mathrm{ZrOCl}_{2} / \mathrm{AgI}\right)$ doped and at $3447 \mathrm{~cm}^{-1}$ in $2: 1\left(\mathrm{ZrOCl}_{2} / \mathrm{AgI}\right)$ doped PANI have been observed. These vibrational bands observed may be explained on the basis of the normal modes of polyaniline. The medium intensity band at $1591 \mathrm{~cm}^{-1}$ (as in undoped PANI) is assigned to the $\mathrm{C}-\mathrm{N}$ stretching of secondary aromatic amine, which shifts to $1518 \mathrm{~cm}^{-1}$ in $2: 1\left(\mathrm{ZrOCl}_{2} / \mathrm{AgI}\right)$ doped samples. The band at $\sim 616 \mathrm{~cm}^{-1}$ observed for undoped and doped PANI samples is the characteristic peak of $\mathrm{C}-\mathrm{H}$ out of plane blending vibration of benzene ring (Grant and Batra 1979; Fink and Leising 1986). On comparing the IR spectra of undoped and doped PANI samples, the medium band observed (in doped samples) around $1586 \mathrm{~cm}^{-1}$ is the characteristic peak of nitrogen quinoid ring and is absent in polyaniline sample. The vibration band at $616 \mathrm{~cm}^{-1}$ is assigned to the benzene ring distribution, whose intensity increases with increase in dopant ratio, 1:1

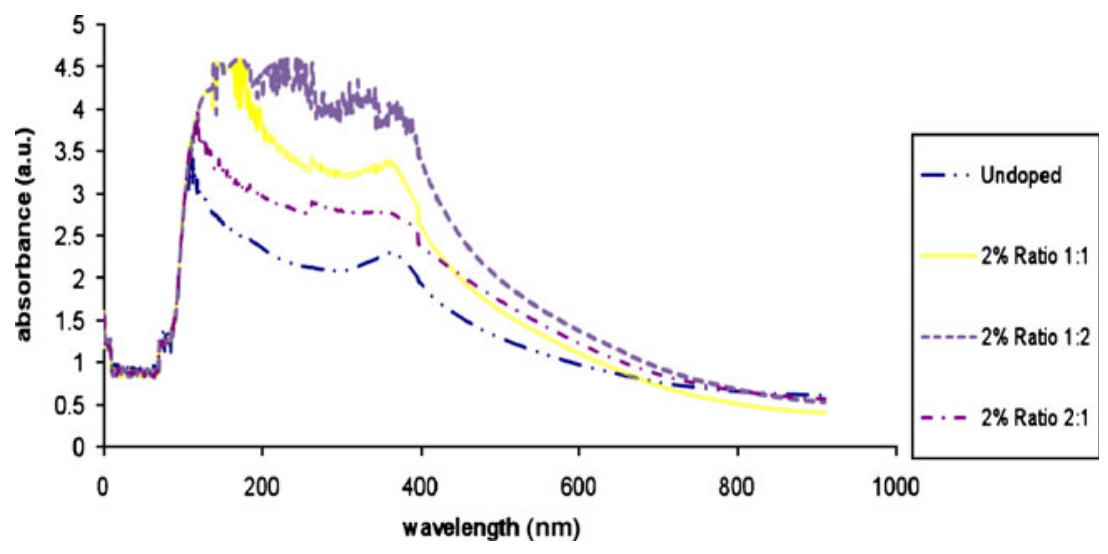

Figure 1. UV-visible absorption spectra of undoped (a) and doped polyaniline at different ratios (b) 1:1, (c) 1:2 and (d) 2:1 (w/w) of $\left(\mathrm{ZrOCl}_{2} / \mathrm{AgI}\right)$. 

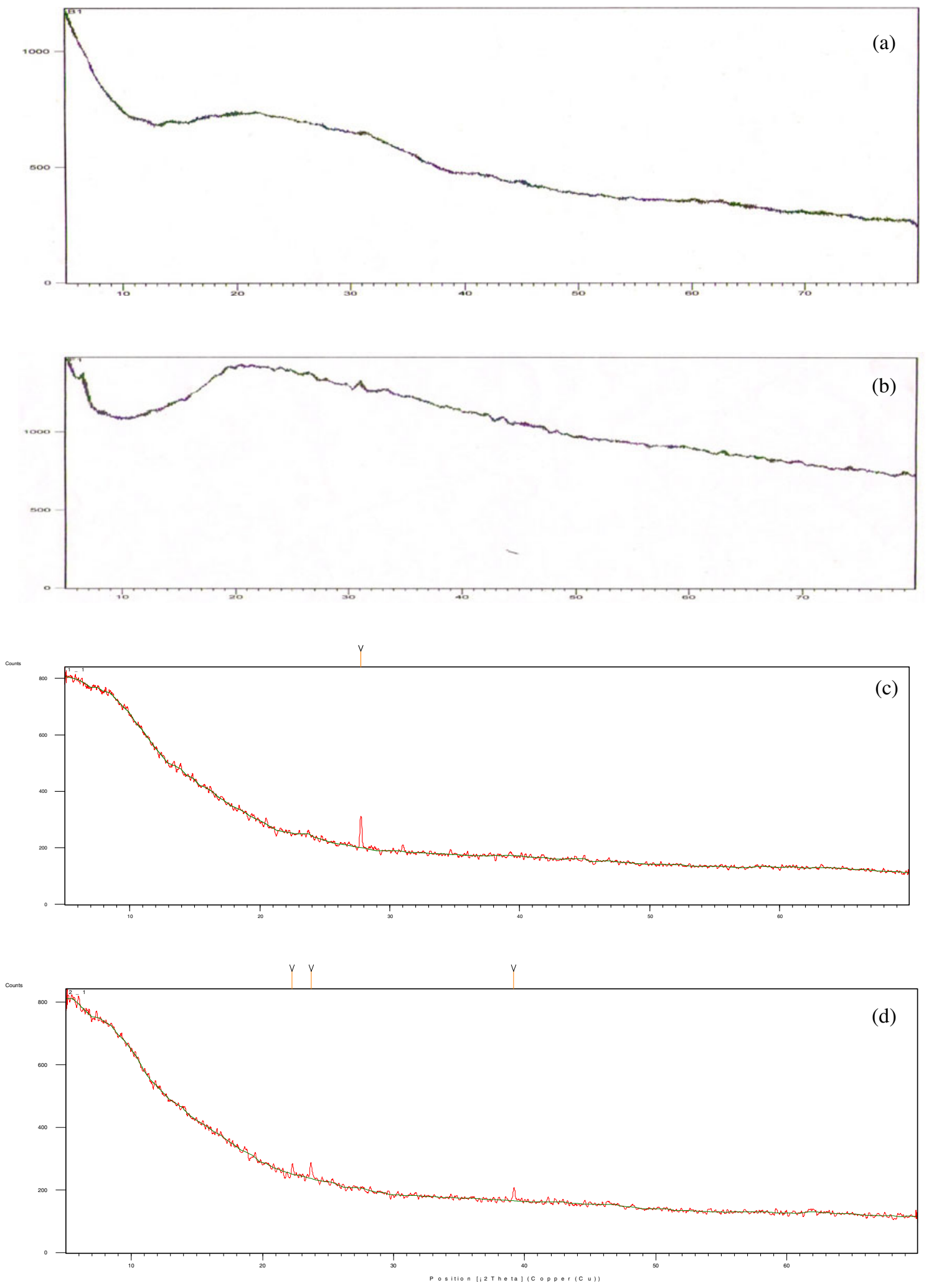

Figure 2. X-ray diffraction patterns of undoped (a) and doped polyaniline at different ratios (b) 1:1, (c) 1:2 and (d) $2: 1(\mathrm{w} / \mathrm{w})$ of $\left(\mathrm{ZrOCl}_{2} / \mathrm{AgI}\right)$. 


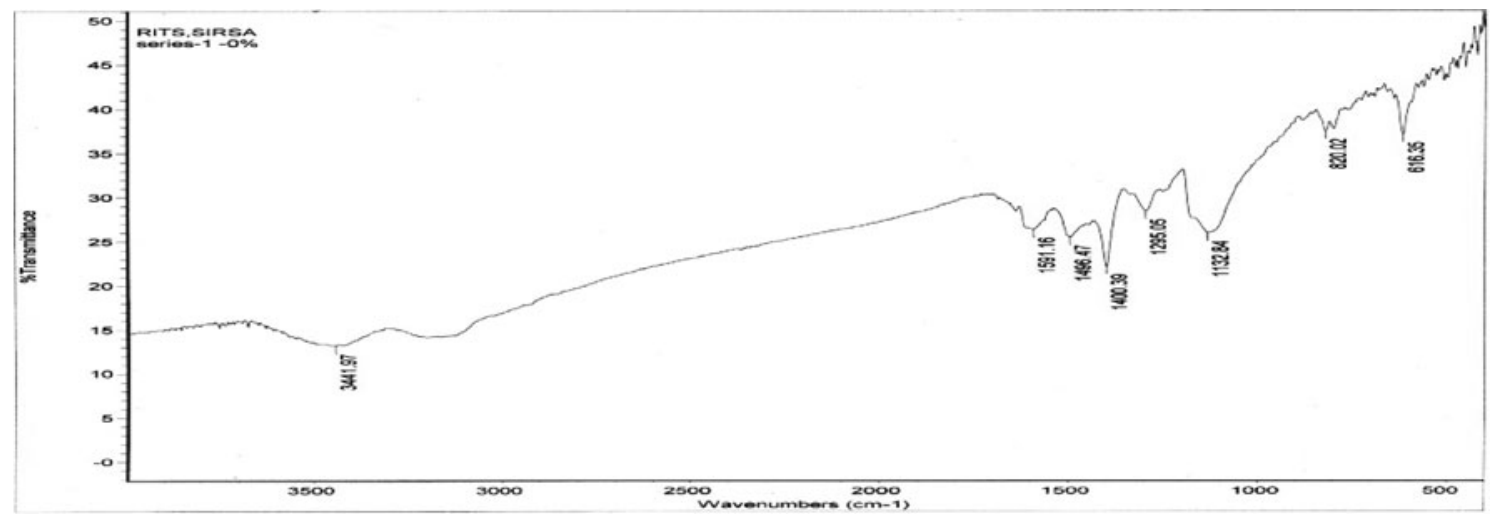

(a)

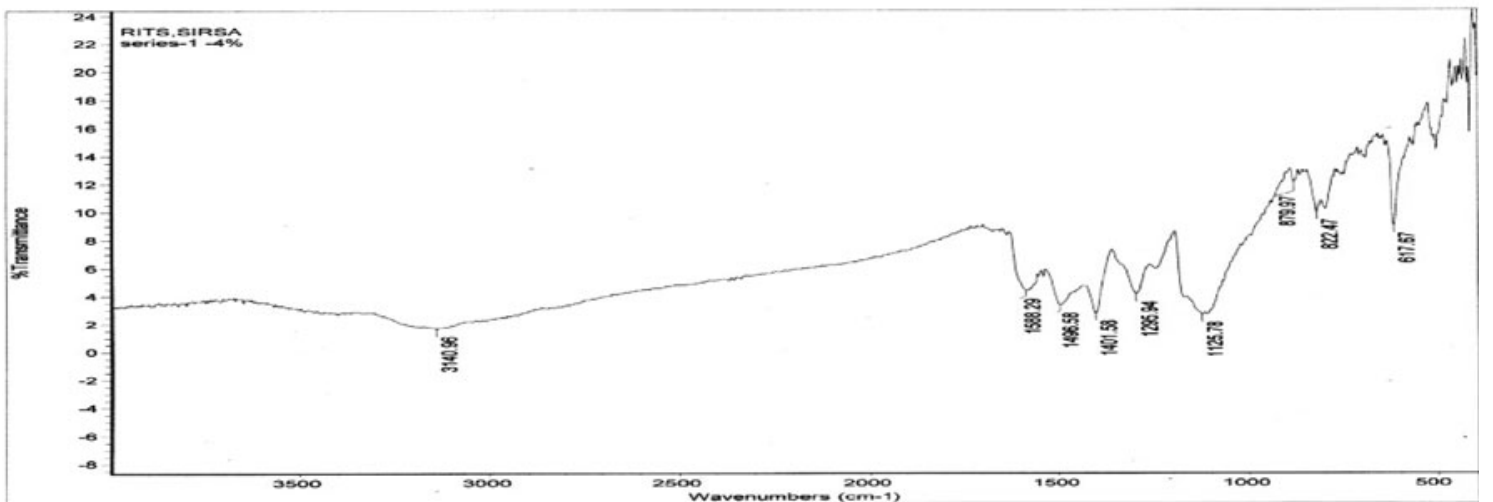

(b)

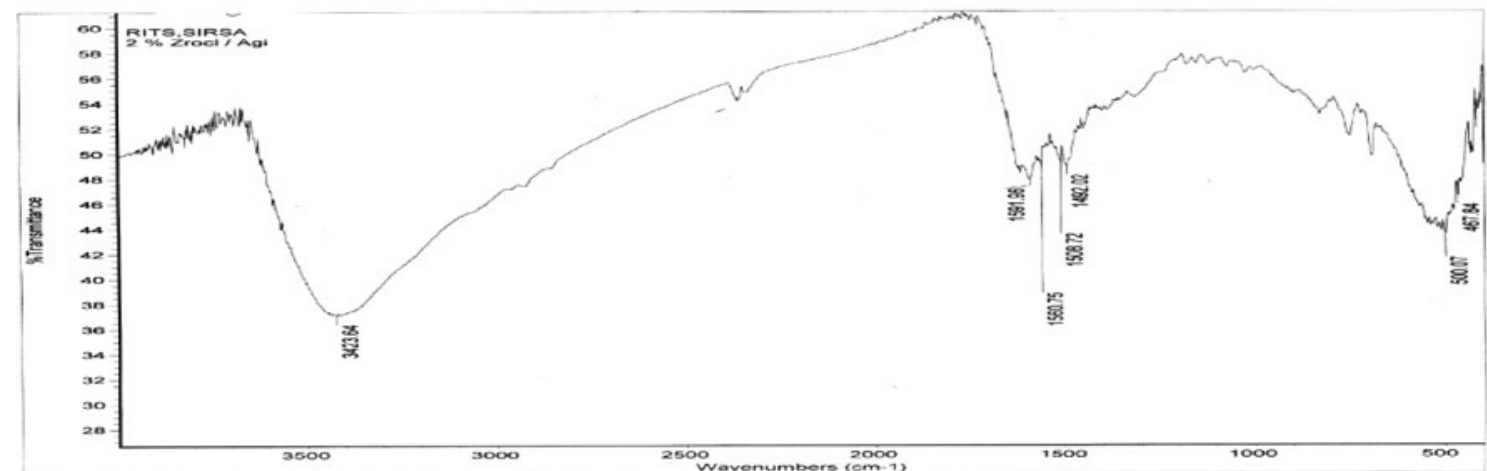

(c)

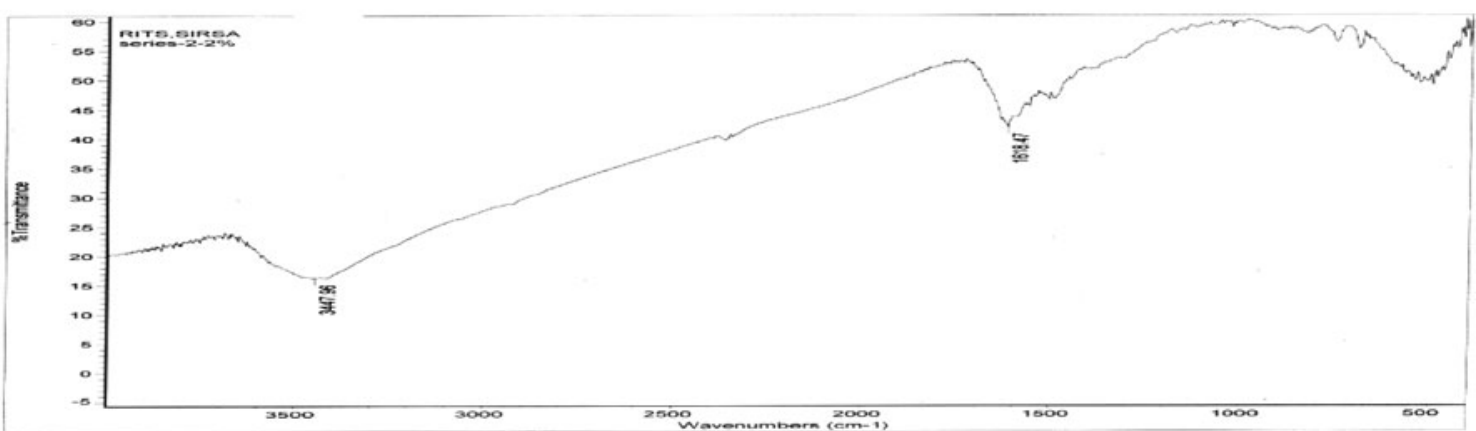

(d)

Figure 3. FTIR spectra of undoped (a) and doped polyaniline with $\mathrm{ZrOCl}_{2} / \mathrm{AgI}$ at different ratios (b) $1: 1$, (c) $1: 2$ and (d) $2: 1(\mathrm{w} / \mathrm{w})$ of $\left(\mathrm{ZrOCl}_{2} / \mathrm{AgI}\right)$. 
$\left(\mathrm{ZrOCl}_{2} / \mathrm{AgI}\right)(\mathrm{w} / \mathrm{w})$. The changes in number and intensity of IR vibrational bands confirmed the dopant interaction with polyaniline.

\subsection{Photoluminescence studies}

The photoluminescence spectroscopy (PL) of $\left(\mathrm{ZrOCl}_{2} / \mathrm{AgI}\right)$ doped PANI has been performed and is shown in figure $4(\mathrm{a}-\mathrm{d})$. It is found that the relative intensity of emission peaks alter with different ratios of binary dopant and nature of solvent (due to polarity). It has been noticed that the peak observed at $442 \mathrm{~nm}$ in undoped PANI shifts towards higher wavelength with change in binary dopant ratio, i.e. 1:1, 1:2 and 2:1. In addition, this peak becomes sharp and intense in the sample having a ratio of $2: 1$ of binary dopant. This may be due to interchain species which plays an important role in the emission process of conjugated polymers. The intensity of peaks depends on factors such as polymer coil size, the nature of polymer-solvent, polymer-dopant

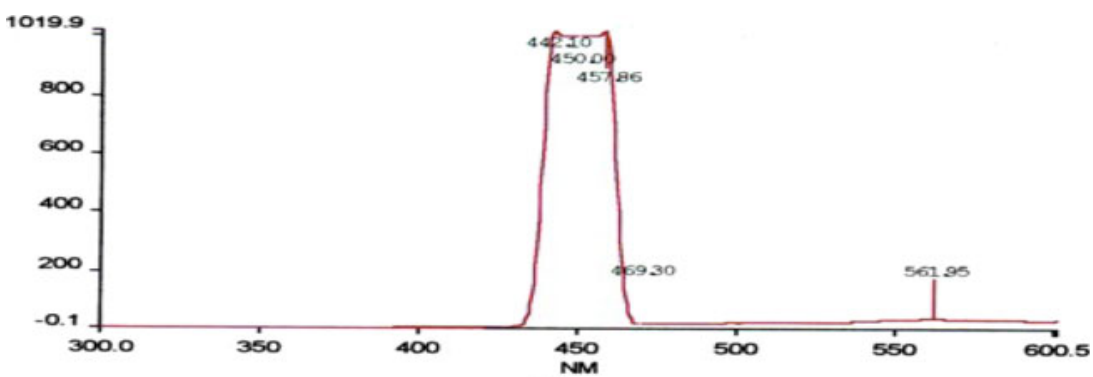

(a)

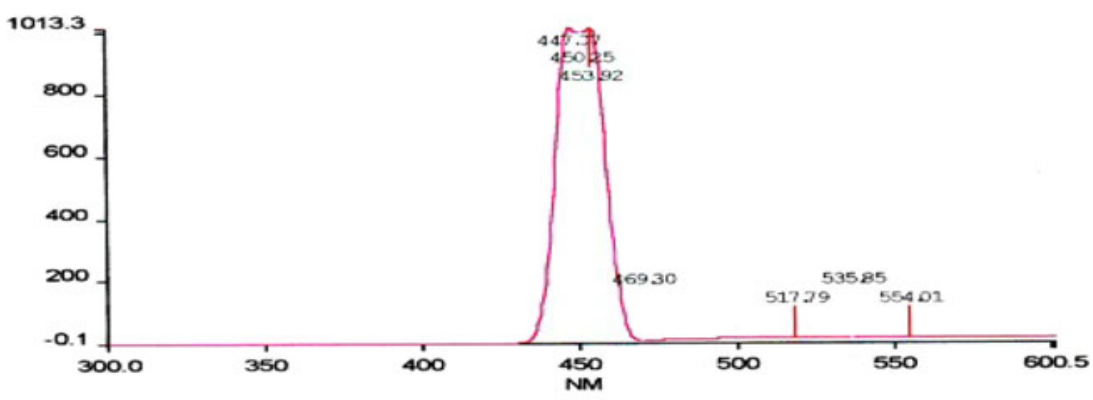

(b)

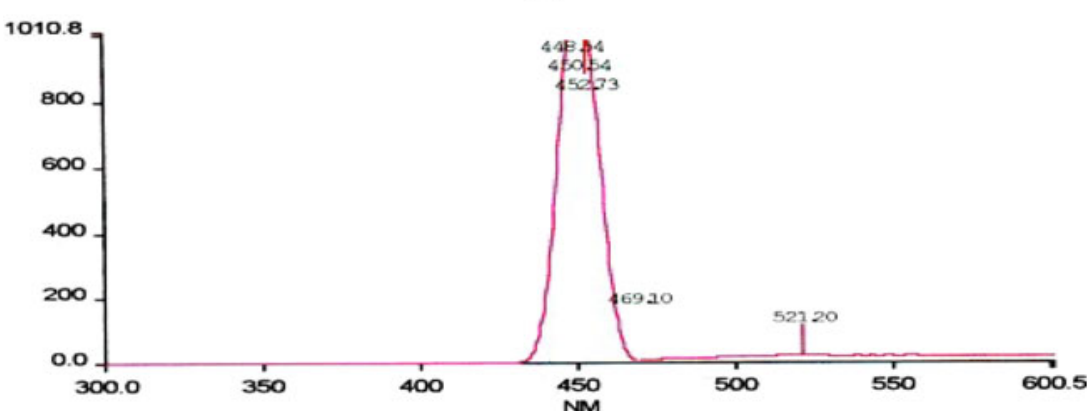

(c)

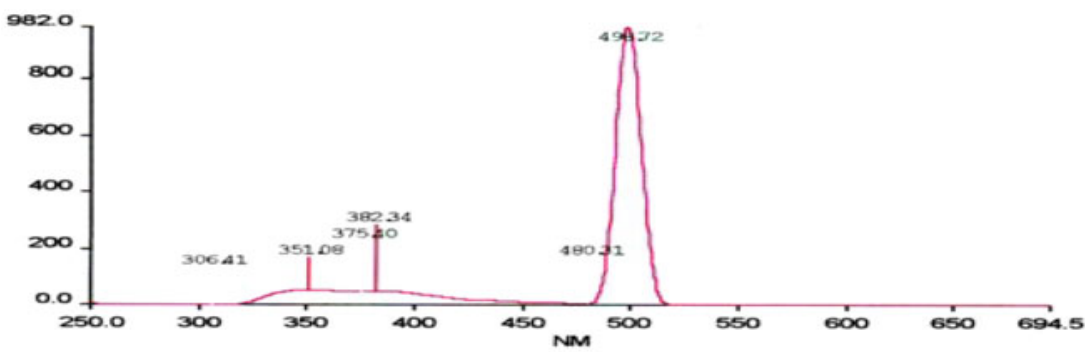

(d)

Figure 4. Photoluminescence spectra of undoped (a) and doped polyaniline at different ratios (b) 1:1, (c) 1:2 and (d) 2:1 (w/w) of $\left(\mathrm{ZrOCl}_{2} / \mathrm{AgI}\right)$. 
interactions, and the degree of chain overlapping (Ameen et al 2007). The PL spectra of samples have the same shape, which indicates that it is an efficient way to tune the intensities of the peak by employing specific dopant with different compositions/ratios.

\section{Conclusions}

The present work describes a significant influence of aqueous binary dopant $\left(\mathrm{ZrOCl}_{2} / \mathrm{AgI}\right)$ in polyaniline for modifying optical and electrical properties. It is interesting to note that the samples are showing both direct and indirect bandgaps, which change with change in dopant's ratio. D.C. conductivity of doped samples is enhanced by three orders. XRD and FTIR spectra of doped PANI indicate the strong interaction of dopant with PANI $\pi$-conjugation system which induces structural modifications in the system. The intensity of emission peaks alter with different dopant ratios and nature of solvent.

\section{Acknowledgements}

Authors wish to express their grateful thanks to Materials Science Lab., Department of Physics, Jamia Millia Islamia, New Delhi; and Punjab University, Chandigarh, for providing experimental facilities.

\section{References}

Ali V et al 2006 J. Phys. Chem. Solids 6768

Ameen S, Ali V, Zulfequar M, Mazharul Haq M and Husain M 2007 J. Polym. Sci. Part B: Polym. Phys. 21265

Epstein A J et al 1994 Synth. Met. 65149

Fink J and Leising G 1986 Phys. Rev. B34 5320

Friend R H, Gymer R W and Holmes A B 1997 Nature 397 121

Gosain D P, Shimizu T, Suzuki M, Bando T and Okano S $1991 \mathrm{~J}$. Mater. Sci. 263271

Grant P M and Batra I 1979 Solid State Commun. 29225

Kazim S, Ali V, Zulfequar M, Haque M M and Husain M 2006 Curr. Appl. Phys. 768

MacDiarmid A G, Mammone R I, Krawczyk J R and Porter S J 1984 Mol. Cryst. Liq. Cryst. 10589

MacDiarmid A G, Chiang J C, Rinchter A F, Somasiri N L D and Epstein A J 1986 Synthesis and characterization of emeraldine oxidation state by elemental analysis (Dordrecht, Holland: Reidel Pub.)

Majeed Khan M A, Zulfequar M, Kumar A and Husain M 2004 Mater. Chem. Phys. 87179

Planes J, Wolter A, Cheguettine Y, Pron A, Genobd F and Nechtschein M 1998 Phys. Rev. B58 7774

Saraswathi R, Gerard M and Malhotra B D 1999 Appl. Polym. Sci. 74145

Scrosati B 1993 Application of electroactive polymers (London: Chapman \& Hall) 